\title{
Extreme Examples of Intraday Variability - Search for Diffractive Scintillation in the Smallest Quasar, PKS 0405-385
}

\author{
Lucyna Kedziora-Chudczer ${ }^{1,3}$, Jean-Pierre Macquart ${ }^{2}$, \\ David L. Jauncey ${ }^{3}$ \\ ${ }^{1}$ Anglo-Australian Observatory, Epping, NSW 1710, Australia \\ ${ }^{2}$ RCfTA, School of Physics, University of Sydney, NSW 2006, Australia \\ ${ }^{3}$ Australia Telescope National Facility, CSIRO, Australia
}

\begin{abstract}
We discuss conditions which have to be satisfied for observable signatures of diffractive scintillation in intraday variable (IDV) sources. We apply this discussion to the quasar PKS 0405-385 which had a scintillating component with angular size of $5 \mu$ as (Kedziora-Chudczer et al. 1997).
\end{abstract}

\section{Introduction}

Interstellar scintillation (ISS) of extragalactic radio sources at $\mathrm{GHz}$ frequencies leads to strong flux density variability on the timescales of days to hours. Scintillation can be strong or weak depending on the properties of interstellar medium (ISM) towards the source and the observing frequency (Narayan 1992). The characteristic length scale of the scattering, at which the changes in phase of incoming radiation are small, is $r_{\text {diff. }}$. For weak scattering $r_{\text {diff }} \gg r_{\mathrm{F}}$, where $r_{\mathrm{F}}=\sqrt{\lambda D / 2 \pi}$ is the Fresnel scale, $D$ is the distance of the scattering screen and $\lambda$ is the wavelength of the radiation. In the case of strong scattering $r_{\text {diff }} \ll r_{\mathrm{F}}$, and refractive and diffractive scintillations can occur.

Diffractive ISS (DISS) show fully modulated, narrowband variability, while refractive ISS (RISS) is characterized by low amplitude, broadband variations over the much longer timescale, $\tau_{\text {ref }}=\tau_{\text {diff }}\left(r_{\mathrm{F}} / r_{\text {diff }}\right)^{2}$. A source of the angular size $\theta_{s}$ exhibits DISS if $\theta_{s} \leq r_{\text {diff }} / D$. Since the characteristic length scale of RISS, $r_{\text {ref }}$ is much larger than $\mathbf{r}_{\text {diff }}$, the limitations on source size are less restrictive and RISS is often observed in extragalactic radio sources (Rickett et al. 1986). On the contrary, DISS has only been observed in pulsars.

Extragalactic IDV radio sources are thought to harbour small, scintillating components, which may show at least partially modulated DISS with fractional bandwidth, $\Delta \nu / \nu \sim\left(r_{\text {diff }} / r_{\mathrm{F}}\right)^{2}$. If the source size is larger than $\theta_{\text {diff }}=r_{\text {diff }} / D$, the amplitude of DISS decreases by $\theta_{\text {diff }} / \theta_{s}$. The timescale of variability is $\tau_{\text {diff }}=D \theta_{s} / v_{\text {ISM }}$, where $v_{\text {ISM }}$ is the transverse velocity of ISM with respect to the line of sight.

\section{Estimates for PKS 0405-385}

We now discuss the possibility of detecting DISS in our existing data for PKS $0405-385$, which contains $15 \%$ of total flux density in the $5 \mu$ as scintillating component. Figure 1 shows the dynamic spectrum of variations in PKS $0405-385$ at four frequencies, where each pixel is residual flux density, $(I-\langle I\rangle) /\langle I\rangle$, averaged over $10 \mathrm{~min}$. In Kedziora-Chudczer et al. (1997) it 
was shown that the broadband variability at 2.5 and $1.4 \mathrm{GHz}$ is consistent with RISS, with a transition wavelength, $\lambda_{0}$ between strong and weak ISS of $\sim 6 \mathrm{~cm}$. If the size of of the scintillating source is the same at all observed frequencies, we expect to see DISS with modulation index, $m_{\text {DISS }} \approx r_{\text {diff }} / D \theta_{s}=0.12(0.27)$ at $1.4(2.5) \mathrm{GHz}$ and with timescale of variability $\sim 2$ hours. However if the size of the scintillating source increases as a function of wavelength, the amplitude of variations will be quenched.

The bandwidth of DISS scales as $\left(\lambda_{0} / \lambda\right)^{4.4}$, and is expected to be $\sim 7$ and $\sim 12 \mathrm{MHz}$ for 1.4 and $2.5 \mathrm{GHz}$ respectively. If the patches of variable flux density in Figure 1 are due to DISS at these frequencies, this suggest that the bandwidth of DISS must be larger than estimated from our simple model. The DISS bandwidth will be increased if the transition wavelength, $\lambda_{0}$ is in fact longer than we assumed in Kedziora-Chudczer et al (1997). For instance, the bandwidth of DISS increases to $100(180) \mathrm{MHz}$ at $1.4(2.5) \mathrm{GHz}$ for $\lambda_{0} \sim 11 \mathrm{~cm}$. Although at this stage we cannot conclude that the variations seen in our dynamic spectrum are due to DISS, the data is sufficiently interesting and encouraging for us to continue our search for DISS.

Acknowledgments. The Australia Telescope is funded by the Commonwealth Government for operation as a national facility by the CSIRO.

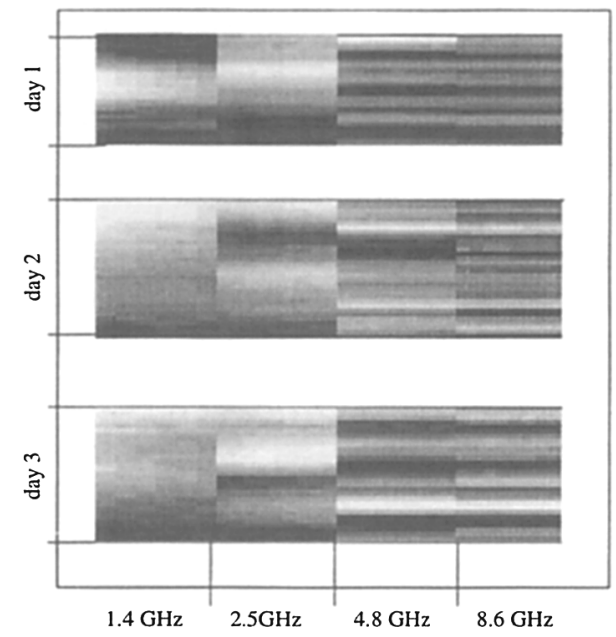

Figure 1. The dynamic spectrum of PKS 0405-385 during the three 12hour observations at four frequencies on the 6, 7 and 8th of June 1996. These are continuum data with $8 \mathrm{MHz}$ wide channels over a bandwidth of $48 \mathrm{MHz}$ at $1.4 \mathrm{GHz}$ and $72 \mathrm{MHz}$ at $2.5,4.8$ and $8.6 \mathrm{GHz}$. The ranges of grey scale are: $(-0.1493,0.1099),(-0.1919,0.1967),(-0.2273,0.3745)$ and $(-0.1859$, $0.3715)$ at $1.4,2.5,4.8$ and $8.6 \mathrm{GHz}$ respectively.

\section{References}

Kedziora-Chudczer, L., Jauncey, D.L., Wieringa, M.H., Walker, M.A., Nicolson, G.D., Reynolds, J.E. \& Tzioumis, A.K., 1997, Ap.J., 490, L9

Narayan, R., 1992, Phil. Trans. R. Soc. Lond., 341, 151

Rickett, B.J., 1986, Ap.J., 307, 564 